\title{
Uniform approximation of planetary ephemerides
}

\author{
J.C. Coma, M. Lara, and T. López Moratalla \\ Real Instituto y Observatorio de la Armada, E-11110 San Fernando, Spain \\ e-mail: teodoro@roa.es
}

Received April 28; accepted September 22, 1997

\begin{abstract}
DE200/LE200 astronomical ephemerides are compressed finding the "best approximation" in the Chebyshev sense. When reproducing the data base from this polynomial approximation, the maxima and minima values of the error are equal in absolute value over the considered interval. In order to obtain polynomials valid also for a reliable evaluation at points interpolated on the data base, we work with dense data bases: the fact that the estimate of the error provided by the best approximation of continuous functions is valid for all the interval and the smoothness of the discrete error functions we have obtained guarantee, in our opinion, the desired bound for the error also for interpolated points. Consequently we obtain maximum compression and fast evaluation.
\end{abstract}

Key words: ephemerides — planets and satellites

\section{Introduction}

Astronomers are supplied with ephemerides coming either from analytical theories or from the numerical integration of the equations of motion. In both cases the astronomical ephemerides are usually provided in tabular form. For points not listed in the tables the user is forced to interpolate. There is another method for providing ephemerides: for any interval of the data base a set of coefficients enables the construction of a polynomial representation from which the user can recover the data base (within a certain precision).

When using polynomial approximations it arises the problem of establishing a bound for the error. For instance, the error coming from a least squares fit varies in a nonuniform way and, hence, it is not possible to make a previous estimation of the error. This inconvenience is avoided when using uniform approximation: the extreme values of the error are equal in absolute value. We describe the method in Sect. 2.

Send offprint requests to: T. López Moratalla
Fitting astronomical ephemerides with polynomial approximations is not a new affair; for instance, since 1980 in "Connaisance des Temps" the Bureau des Longitudes computes series expansions in Chebyshev polynomials representing their analytical theories. Alternative procedures to compress planetary and satellite ephemerides keeping a good precision have also been proposed (Chapront \& Vu 1984; Chapront 1995).

When an analytical function is approximated by means of a polynomial, fast evaluation is ensured for any point of the interval of validity. The approximation of discrete functions is a different matter. At first one should only claim to recover the data base from the polynomial approximation but in many cases it can be also used for interpolation between points of the data base.

In this work we approximate discrete functions finding the best approximation in the Chebyshev sense. We apply this uniform approximation to the Jet Propulsion Laboratory DE200/LE200 planetary and lunar ephemerides (Newhall et al. 1983). It must be mentioned that the Nautical Almanac Office of the U.S. Naval Observatory issued the "Almanac for Computers" from 1977 (Kaplan et al. 1977) to 1991, where standard Chebyshev approximation was used to compute polynomials covering specified intervals of time (Kaplan, private communication). These polynomial approximations are used to produce ephemerides with lower precision than DE200/LE200. Beyond 1991 that publication was replaced by integrated packages of software and astronomical data.

In our approach using the best approximation we obtained medium or low degree polynomial valid for long time intervals except for the moon. In Sect. 3 comparisons with standard Chebyshev approximation and least squares fit are carried out. Best results were always on the side of the best approximation.

In Sect. 4 the best approximation is used for construction of the ANDI (Lara \& López 1997), an electronical nautical almanac where fast evaluation is required. In order to overcome the inconvenience that the estimator of the error is only valid for the set of points used to construct 
the approximation, we look back to the best approximation to continuous functions, where the estimate for the error fits for all the interval of validity. Working with dense data bases, the smoothness of the resulting discrete error functions guarantees the reliable use of the approximating polynomials like interpolating ones.

\section{The best polynomial approximation}

In what follows we will refer to the interval $-1 \leq x \leq 1$. There is not lack of generality, for projecting any closed interval $[a, b]$ onto the interval $[-1,1]$ is a trivial problem.

When using a Lagrange polynomial $P_{n}(x)$ of degree $n$ for interpolating a continuous function $f(x)$ we get

$f(x)=P_{n}(x)+E_{n}(x)$.

The function $E_{n}(x)$ is the Lagrange remainder given by

$E_{n}(x)=Q(x) \frac{f^{(n+1)}(\xi(x))}{(n+1) !}$,

where $Q(x)$ is the polynomial of degree $n+1$

$Q(x)=\left(x-x_{0}\right)\left(x-x_{1}\right) \ldots\left(x-x_{n}\right)$.

It is well known that he maximum absolute value of $Q(x)$ is minimum when the abscissas $x_{0}, x_{1}, \ldots, x_{n}$ are chosen to be the zeros of the Chebyshev polynomial $T_{n+1}(x)$ of degree $n+1$. Nevertheless, this polynomial could be not the best approximation to the function $f(x)$. One will succeed with the best approximation when it is achieved in Eq. (1) the minimum value of $\left|E_{n}\right|$ over the entire interval. Therefore, we will say that the polynomial $P_{n}^{*}(x)$ is the best approximation of degree $n$ to the function $f(x)$ when

$$
\max _{-1 \leq x \leq 1}\left|P_{n}^{*}(x)-f(x)\right| \leq \max _{-1 \leq x \leq 1}\left|P_{n}(x)-f(x)\right| .
$$

An important property characterizes the best approximation: at least at $n+2$ critical points of the interval $[-1,1]$ the error function $E(x)=P_{n}^{*}(x)-f(x)$ assumes extremes values that are equal in magnitude and alternating in sign (see, for instance, Vallée-Poussin 1989). From this property, if a set $x_{1}<x_{2}<\ldots<x_{n+2}$ of critical points is known it is clear that $P_{n}^{*}(x)$ is easily determined from the $n+2$ linear equations

$$
P_{n}^{*}\left(x_{k}\right)-f\left(x_{k}\right)=(-1)^{k-1} \eta, \quad(k=1, \ldots, n+2),
$$

in the unknowns $\eta$ and the $n+1$ coefficients of $P_{n}^{*}(x)$. Consequently the problem of computing the best approximation to a function $f(x)$ can be reduced to determine the set $\left(x_{0}, x_{1}, \ldots, x_{n+1}\right)$ of $n+2$ points where

$\max \left|P_{n}^{*}(x)-f(x)\right|=\left|P_{n}^{*}\left(x_{k}\right)-f\left(x_{k}\right)\right| \quad(0 \leq k \leq n+1)$.

The critical points are not known a priori, but starting from a set of references points close enough to the critical one we can proceed by iterations to compute the polynomial coefficients. Murnaghan \& Wrench (1959) assume the convergence of the iterations and find the best approximation by changing the complete set of abscissas at each step. Without need of this assumption, Stiefel (1958) demonstrated that, when proceeding by interchange in the transition from one set of reference points to the next, the iteration takes place in a finite number of steps.

Basically the procedure consists of the following steps:

1. select a trial set of reference points

2. compute the set of coefficients of the polynomial approximation

3. check that it is the best approximation

4. if not, change the set of reference points and come back to step 2 .

Assuming that we can approximate the astronomical ephemerides by truncated series of Chebyshev polynomials, the error in their best approximation is in most cases comparable to the first of the neglected terms. Consequently the zeros of the derivative of the Chebyshev polynomial of degree $n+1$ can be selected at step 1 as a first approach to the reference points (Deprit et al. 1945).

The procedure is different when approximating a function given by a finite set of $m$ points $f\left(x_{i}\right),(1 \leq i \leq m)$. Since we want to compute a polynomial of degree $n$ which fits the data, we face up to an undetermined system: $m$ points and $n$ coefficients $(n<m)$. The direct approach to the problem is to solve the inconsistent system

$\sum_{0 \leq j \leq n} x_{i}^{j} c_{j}+f\left(x_{i}\right)=\eta_{i} \quad(i=0, \ldots, n+1)$

of $n+2$ linear equations where the solution $c_{j}$ (the coefficients of the polynomial) minimizes $\max \left|\eta_{i}\right| \quad(0 \leq i \leq$ $n+1)$. Then, the computation of the best approximation consists in determining the set of coefficients $c_{j}$ which minimizes

$\max \left|f\left(x_{i}\right)-\sum_{0 \leq j \leq n} c_{j} x_{i}^{j}\right| \quad(1 \leq i \leq m)$.

The problem can be attacked in two ways. The first one begins with an initial estimation of the coefficients and uses an algorithm that tries to diminish the error function $\left|P_{n}(x)-f(x)\right|$ at each step. The other, consists in finding the set of reference points $x_{k}$ that maximize $\mid P_{n}\left(x_{k}\right)-$ $f\left(x_{k}\right)$. Stiefel (1960) demonstrated that both methods are equivalent to the simplex method of linear programming.

In our work we construct the approximating polynomials by using the Stiefel exchange-method (Stiefel 1958). A set of $n+2$ points is selected as reference points from the set of $m$. The starter reference points are those with closest abscissas to the zeros of the derivative with respect to $x$ of the Chebyshev polynomial $T_{n+1}$, plus both ends \pm 1 . The exchange-method is an iterative one that, by changing the reference points, computes those coefficients $c_{j}$ such that for $i=1$ to $m$ they minimize Eq. (2). 
Uniform approximation is ensured when Remez's rippling occurs (Remez 1957), i.e. the errors at reference points are equal in absolute value but opposite in sign. When this situation occurs the polynomial obtained is optimal.

The Stiefel exchange-method has been implemented by Barrodale (1975) and Schmitt (1971). Deprit \& Picard (1979) used the Barrodale algorithm with encouraging results. In our work we first used the Barrodale algorithm but the Schmitt one showed better results.

\section{The best approximation and other polynomial approximations}

The computation of the best approximation is much more laborious than the simple computation of the LagrangeChebyshev polynomial. In practice, in many problems it could be not justified the extra job of computing the best approximation because of the small increase of accuracy (Clenshaw 1964). Nevertheless, in reference to planetary ephemerides we believe that it is not the case. In order to justify this assertion, in this section we present comparisons between the best approximation and other approximation methods.

First we compare the best approximation with standard Chebyshev approximation. The comparisons have been made for the Sun and navigational planets between the series provided by the "Almanac for Computers" and the best approximation computed for the same precision and interval. In this publication the series were obtained by computing standard Chebyshev approximations to the data published in "The Astronomical Almanac". The comparisons have been carried out using the values listed in the Astronomical Tables (Section D) of "Almanac for Computers 1989" (hereafter termed AC89).

Table 1 presents the results for high precision series valid for intervals of 95 days. Using uniform approximation we always obtained series shorter than those provided in AC89 for the same precision. It is remarkable the case of Venus where the number of terms is drastically reduced. Analogous results are showed in Table 2 for low precision series. The column "Maximum error" of both tables corresponds to the values given in pages A9 and A11 of the Explanation of the AC89 with two exceptions for Table 2:

For the distance of Venus we present the error obtained with the 30 terms of the best approximation, the same degree as used for the other coordinates; although that precision is slightly worse than the one listed in AC89, it is indeed enough for a low precision series. The second exception is for the declination of Saturn, where we checked that the real value of the error $\left(0.66^{\prime \prime}\right)$ is slightly larger than the estimation given in the AC89 (0.4").

Finally, we present a comparison between least squares fit and the best approximation. Least squares fit is not an uniform approximation: the error function oscillates with nonuniform magnitude, being normally minimal in the center of the interval. In order to illustrate this well known
Table 1. High precision series. The span of validity is 95 days. We note LS for the number of terms of the polynomial approximation when using least squares fit and BA for the best approximation. "au" stands for astronomical units

\begin{tabular}{lllll}
\hline Body & & LS & BA & Maximum error \\
\hline Sun & Right Ascension & 24 & 21 & 0.01 \\
& Declination & 24 & 21 & $0^{\prime \prime} .01$ \\
& Distance & 24 & 21 & $410^{-7}$ au \\
Venus & Right Ascension & 30 & 20 & 0.01 \\
& Declination & 30 & 20 & $0^{\prime \prime} 1$ \\
& Distance & 30 & 20 & $110^{-6}$ au \\
Mars & Right Ascension & 16 & 15 & 0.03 \\
& Declination & 16 & 15 & $0^{\prime \prime} \cdot 2$ \\
& Distance & 16 & 15 & $110^{-6}$ au \\
Jupiter & Right Ascension & 16 & 14 & 0.03 \\
& Declination & 16 & 14 & $0^{\prime \prime} 1$ \\
& Distance & 16 & 14 & $210^{-6}$ au \\
Saturn & Right Ascension & 12 & 11 & 0.04 \\
& Declination & 12 & 11 & $0^{\prime \prime} \cdot 4$ \\
& Distance & 12 & 11 & $110^{-5}$ au \\
\hline
\end{tabular}

effect we present a concrete example using both techniques. We have computed approximating polynomials of degree 4 covering 32 days of the right ascension of Jupiter for June 1997. The comparisons have been made computing the coefficients of ordinary and Chebyshev polynomials. Figure 1 shows the error functions in both cases. In the above plot we used least squares fit with ordinary polynomials; in the other plot Chebyshev polynomials are used. Notice that the largest errors of the least squares fit occur in both cases at the ends of the interval. The least squares fit with Chebyshev polynomials resulted in a very good approximation, but notice also that one should reject both ends of the interval for providing an accuracy similar to the one reached by the best approximation. The uniform behavior of the best approximation implies that there is no need of taking slopes.

With the best approximation a rigorous estimate of the error valid for the entire interval is directly provided by the method. This is not the case of least squares fit, but in practical applications it is not a major problem: an estimate of the error can be always computed a posteriori making difference between source and approximation data.

\section{Planetary Ephemerides for Navigators}

The practical application of the previous results has been the implementation of the ANDI, a computer application providing astronomical data for navigators. For the selection of the ephemerides representation the following considerations are in order: on one hand truncated series 
Table 2. Low precision series. The span of validity is 366 days. We note LS for the number of terms of the polynomial approximation when using least squares fit and BA for the best approximation. "au" stands for astronomical units

\begin{tabular}{|c|c|c|c|c|}
\hline Body & & $\mathrm{LS}$ & $\mathrm{BA}$ & Maximum error \\
\hline Sun & $\begin{array}{l}\text { Right Ascension } \\
\text { Declination } \\
\text { Distance }\end{array}$ & $\begin{array}{l}22 \\
22 \\
22\end{array}$ & $\begin{array}{l}18 \\
18 \\
18\end{array}$ & $\begin{array}{l}0.5 \\
3^{\prime \prime} \\
410^{-5} \text { au }\end{array}$ \\
\hline Venus & $\begin{array}{l}\text { Right Ascension } \\
\text { Declination } \\
\text { Distance }\end{array}$ & $\begin{array}{l}50 \\
50 \\
50\end{array}$ & $\begin{array}{l}30 \\
30 \\
30\end{array}$ & $\begin{array}{l}0.3 \\
3^{\prime \prime} \\
310^{-5} \text { au }\end{array}$ \\
\hline Mars & $\begin{array}{l}\text { Right Ascension } \\
\text { Declination } \\
\text { Distance }\end{array}$ & $\begin{array}{l}14 \\
14 \\
14\end{array}$ & $\begin{array}{l}11 \\
11 \\
11\end{array}$ & $\begin{array}{l}1^{\mathrm{s}} \\
8^{\prime \prime} \\
410^{-5} \mathrm{au}\end{array}$ \\
\hline Jupiter & $\begin{array}{l}\text { Right Ascension } \\
\text { Declination } \\
\text { Distance }\end{array}$ & $\begin{array}{l}14 \\
14 \\
14\end{array}$ & $\begin{array}{l}14 \\
14 \\
14\end{array}$ & $\begin{array}{l}0.1 \\
00^{\prime \prime} 7 \\
410^{-5} \text { au }\end{array}$ \\
\hline Saturn & $\begin{array}{l}\text { Right Ascension } \\
\text { Declination } \\
\text { Distance }\end{array}$ & $\begin{array}{l}14 \\
14 \\
14\end{array}$ & $\begin{array}{l}14 \\
14 \\
14\end{array}$ & $\begin{array}{l}0.1 \\
0^{\prime \prime} .7 \\
410^{-5} \text { au }\end{array}$ \\
\hline
\end{tabular}

\section{Arc seconds}

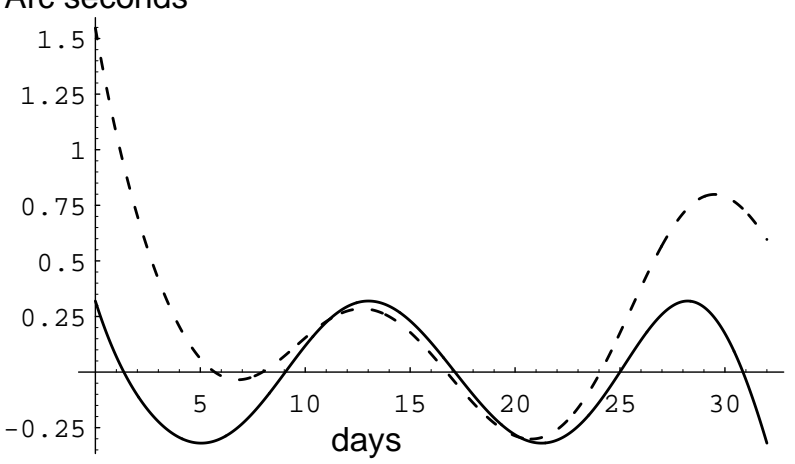

Arc seconds

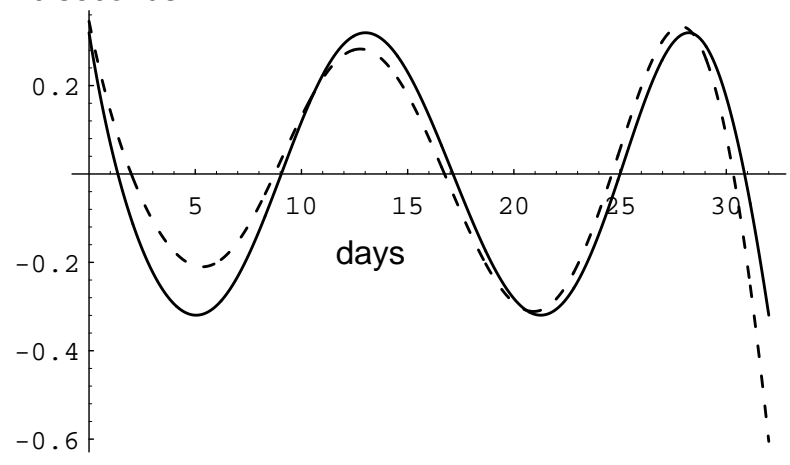

for planetary motion comprise large spans, on the other hand polynomial approximations have the advantage of its simple and fast evaluation, what is rather convenient due to the iterative procedures carried out during the program execution.

A precision better than 0.1 arc minutes is required for navigational purposes. Despite of this low value the precision requirement could be unfulfilled using truncated series for planetary motion; thus, in "Planetary Programs and Tables" (Bretagnon \& Simon 1956) the accuracy is bounded to 0.01 . That circumstance and the fact that the computation of certain critical phenomena could spent an appreciable time, convinced us to use polynomial approximations; and because of the results of Sect. 3 we decided to use the best approximation method.

Again, we based our computations on DE200/LE200 basic ephemerides. By properly corrections of precession, nutation and aberration, apparent coordinates are obtained. Then the coefficients of all necessary polynomials are computed using the Schmitt algorithm. For a desired error the polynomial degree and the amplitude of the interval cannot be selected in an arbitrary manner. From practical considerations we decided to use medium or low degree polynomials and, except for the moon, we selected an interval of a year when possible; otherwise the interval was reduced to half year. Table 3 presents results corresponding to 1997 . In order to use the computed polynomial approximations like interpolating polynomials it was enough to work with data bases tabulated every 4 hours except for the moon; in this last case we used a much more dense data base tabulated every 20 minutes.

Table 3. Degrees of approximating polynomials. Fixing an error of 0.05 arc minutes we use different time spans depending on the body. Polynomials corresponding to 1997

\begin{tabular}{llrc}
\hline Body & & days & degree \\
\hline Sun & Right Ascension & 183 & 19 \\
& Declination & 365 & 16 \\
Moon & Right Ascension & 31 & 19 \\
& Declination & 31 & 18 \\
Venus & Right Ascension & 183 & 18 \\
& Declination & 365 & 21 \\
\multirow{4}{*}{ Mars } & Right Ascension & 183 & 22 \\
& Declination & 365 & 24 \\
\multirow{4}{*}{ Jupiter } & Right Ascension & 365 & 15 \\
& Declination & 365 & 14 \\
\multirow{2}{*}{ Saturn } & Right Ascension & 365 & 12 \\
& Declination & 365 & 10 \\
\hline
\end{tabular}

Fig. 1. Error functions for the right ascension of Jupiter. Ordinary (above) and Chebyshev (below) polynomials of degree 4 approximating 32 days of 1997. Dashed line corresponds to least squares fit while full line is the best approximation
Figure 2 shows the error functions for the right ascension and declination of the Sun and Mars. For the 

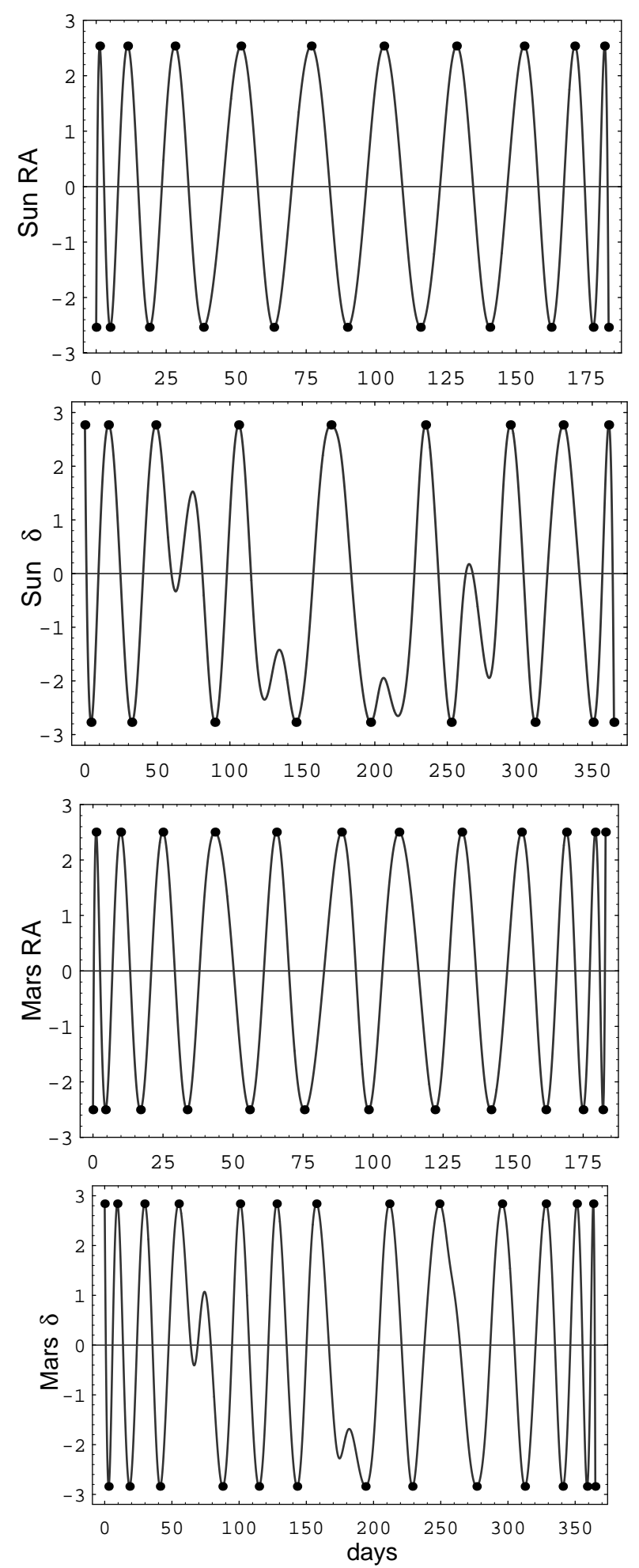

Fig. 2. Error functions for the right ascension (RA) and declination $(\delta)$ of the Sun (above) and Mars (below). Time intervals and polynomial degrees correspond to the values presented in Table 3. Ordinates are arc seconds. Black dots correspond to errors in critical points right ascensions the time span is 183 days and for the declinations 365 days. Polynomial degrees are detailed in Table 3. Notice the alternance in sign of the extreme values predicted by Remez (1957). Though the error functions for declinations are not as smooth as for right ascensions, notice again the alternance of sign in critical points (black dots). Uniform approximation is ensured in all cases. From the smoothness of the error functions we conclude that the error has also the same bound for interpolated points of the data base and consequently the approximating polynomials can also be used for fast evaluation at any point.

\section{Conclusions}

Planetary ephemerides have been compressed using the best approximation in the Chebyshev sense. Using this uniform approximation we have got better compression than the one obtained when using other approximation procedures.

The application of the Schmitt algorithm to DE200/LE200 showed encouraging results: in each case the data base is recovered within the bound for the error provided by the algorithm. The approximating polynomials obtained can also be used for fast evaluation at interpolated points, but for the moon we were forced to deal with a dense data base.

Application of this uniform approximation to compact calculus of "Almanaque Náutico" showed very reliable. This method will be implemented as part of the navigation system of the Spanish Navy.

Acknowledgements. We thank Dr. Deprit who suggested us the application of minimax methods to the compression of astronomical ephemerides. Thanks are due to Dr. Chapront for many comments and suggestions while acting as a referee. This work has been supported in part by the Spanish Ministry of Education (DGICYT Project \# PB96-0522).

\section{References}

Almanac for Computers, U.S. Naval Observatory

Almanaque Náutico para uso de los navegantes, Real Instituto y Observatorio de la Armada

Barrodale C.P., 1975, ACM Trans. Math. Software 1, 264

Bretagnon P., Simon J-L., 1986, Planetary Programs and Tables from -4000 to +2800 , Willmann-Bell, Inc., Richmond, Virginia

Chapront J., 1995, A\&AS 109, 181

Chapront J., Vu D.T., 1984, A\&A 141, 131

Clenshaw C.W., 1964, J. SIAM Numer. Anal., Ser. B 1, 26

Connaissance des Temps, Bureau des Longitudes

Deprit A. Picard H., 1979, NRL, Report 8280

Deprit A., Poplarchek W., Deprit-Bartholomé A., 1975, Celest. Mec. 11, 53

Kaplan G.H., Dogget L.E., Seidelmann P.K., 1976, U.S. Naval Observatory Circular No. 155

Lara M., López T., 1997, ANDI-Almanaque Náutico en disquete, Boletín ROA in preparation, Real Instituto y Observatorio de la Armada 
Murnaghan F.D., Wrench J.W., 1959, Maths. of Comp. 13, 185 Newhall X.X., Standish E.M., Williams J.G., 1983, A\&A 125, 150

Remez E., 1957, General Computation Methods for Chebyshev Approximation, Izdat. Akad. Nauk. Ukranisk SSR, Kiev Schmitt H., 1971, Comm. ACM 14, 355

Stiefel E.L., 1958, in: Numerical Approximation, Langer R. (ed.), U. Winsconsin, p. 217

Stiefel E., 1960, Numerische Mathematik 2, 1

The Astronomical Almanac, U.S. Naval Observatory and Royal Greenwich Observatory

Valleé-Poussin C. de la, 1919, Leçons sur l'approximation des fonctions d'une variable réelle. Paris Gauthier-Villars 